\title{
A evolução do significado atribuído ao acrônimo BIM: Uma perspectiva no tempo
} The evolution of the meaning ascribed to the acronym BIM: A perspective in time

\author{
João Alberto da Motta Gaspar \\ Universidade de Campinas \\ 192355@unicamp.br \\ Regina Coeli Ruschel \\ Universidade de Campinas \\ ruschel@fec.unicamp.br
}

\begin{abstract}
The term Building Information Model emerged in 1992. It has evolved over time and has its meaning currently associated with an object-oriented modeling technology and an associated set of processes to produce, communicate and analyze building models. Its origin is related to several other, older terms. This paper registers the evolution of BIM and related definitions over time by means of a systematic literature review. We present a list of BIM-related terms and their meanings, organized by date of emergence, and charts showing which ones are most used over time, contributing to better understanding of the meaning of BIM.
\end{abstract}

Keywords: BIM; History of BIM; Building Information Model.

\section{Introdução}

Succar (2009) faz considerações a respeito do significado do termo BIM à época em que foi publicado e aponta as dificuldades relacionadas à compreensão de seu sentido. $\mathrm{Na}$ introdução de seu texto, o autor afirma que:

"Mesmo que o termo BIM sofra contestações a respeito de sua utilidade, continua a ser utilizado pela academia e pela indústria como o 'novo paradigma CAD'” (tradução nossa).

Mais adiante, Succar aponta a existência de diversos termos utilizados pela academia e indústria que têm a mesma conotação do termo BIM, os quais, segundo ele, são "amplamente utilizados", sem, contudo, apresentar critérios objetivos para a inclusão de um ou outro termo na lista.

A partir dessa leitura, foi possível assumir que pesquisar a respeito da evolução do significado do termo BIM ao longo de sua história pode representar uma importante contribuição. Foi esse, portanto, o ponto de partida para o início deste estudo. A pesquisa, porém, não poderia começar apenas pela investigação dos termos apresentados pela tabela de Succar. Foi preciso buscar mais termos preliminares para investigação antes de uma pesquisa mais aprofundada. Para tanto, optou-se por fazer um levantamento sobre "história do BIM" pelo mecanismo de busca do Google.

Após avaliação dos resultados encontrados, percebeu-se que a maior parte dos textos era uma adaptação (ou cópia, com citação expressa ou não) de um artigo do arquiteto Michael Bergin, mestre em Computational Design pela University of California em Berkeley e professor da mesma instituição entre 2011 e 2015. Além da questão relacionada à reincidência da fonte, o que pode ser um indício da relevância do texto de
Bergin, foi possível constatar que ele é o único que justifica seu ponto referencial sobre as origens do BIM a partir de um método que pode ser reproduzido, qual seja, a extração de significado de um termo em um artigo produzido por pesquisadores com reconhecimento passível de ser medido por meio da quantidade de citações. Em artigo postado em seu blog, Bergin (2011) afirma que:

"The conceptual underpinnings of the BIM system go back to the earliest days of computing. As early as 1962, Douglas C. Englebart gives us an uncanny vision of the future architect in his paper Augmenting Human Intellect. "the architect next begins to enter a series of specifications and data - a six-inch slab floor, twelve-inch concrete walls eight feet height within the excavation, and so on. When he has finished, the revised scene appears on the screen. A structure is taking shape. He examines it, adjusts it... These lists grow into an evermoredetailed, interlinked structure, which represents the maturing thought behind the actual design."

Ao apresentar o trabalho de Engelbart, pesquisador de Stanford (pioneiro da computação moderna e referência para outros pioneiros da computação aplicada a projetos, como Ivan Sutherland), o autor termina por extrair os termos que subsidiam sua pesquisa histórica sobre BIM, e que também se tornaram a primeira referência para esta pesquisa: a) projeto orientado a objetos (object-based design), b) manipulação paramétrica (parametric manipulation), e c) bancos de dados relacionais (relational database). A partir de então, Bergin começa a relatar sua versão da história do BIM, utilizando como principal referência bibliográfica o livro Manual de BIM - 1aㅡ Edição (Eastman et al., 2008), de onde foi retirado o significado do termo BIM para fins de controle para esta pesquisa. Nesse livro, tido como a referência pela academia e mercado, BIM é definido como: 
"uma tecnologia de modelagem e um conjunto associado de processos para produzir, comunicar e analisar modelos de construção".

Comparando essa definição com a descrição do processo de produção automatizada de projetos feita por Engelbart em 1962, é possível perceber as semelhanças entre os dois pesquisadores acerca do entendimento de que a associação entre tecnologias e processos é o marco de um novo paradigma na forma de projetar, mesmo que entre os dois textos tenham se passado 46 anos. Essa similaridade também foi levada em consideração para validar o entendimento de Bergin sobre o texto de Engelbart como o ponto de início da pesquisa sobre os termos que, historicamente, trazem consigo o mesmo significado BIM de referência, definido por Eastman et al. (2008).

Desta forma, o objetivo deste estudo é registrar a evolução do termo BIM ao longo de sua história, assim como seu significado. Para tanto, adotou-se o método da Revisão Sistemática da Literatura. Os termos utilizados nesta revisão foram identificados preliminarmente a partir das leituras de Engelbart (1962), Eastman et al. (2008) e Bergin (2011) e, posteriormente, a partir dos resultados encontrados já no momento da busca dentro das bases de dados acadêmicas. A Tabela 1 apresenta os termos referenciais iniciais (históricos e de controle), assim como os termos pesquisados.

Tabela 1: Termos utilizados na pesquisa, suas origens e funções. Fonte: Autores.

\begin{tabular}{|c|c|}
\hline Termos utilizados na pesquisa & $\begin{array}{l}\text { Origem e função no } \\
\text { contexto da pesquisa }\end{array}$ \\
\hline $\begin{array}{l}\text { Object-based Design } \\
\text { Parametric Manipulation } \\
\text { Relational Database }\end{array}$ & $\begin{array}{l}\text { Bergin (2011) a partir de } \\
\text { Engelbart (1962): } \\
\text { termos de referência para } \\
\text { identificar outros termos que } \\
\text { podem ter relação de } \\
\text { significado com o termo BIM } \\
\text { para fins de controle. }\end{array}$ \\
\hline BIM (Building Information Model) & $\begin{array}{l}\text { Eastman et al. (2008): } \\
\text { termo contemporâneo, que } \\
\text { dá significado a BIM, para } \\
\text { controle da pesquisa. }\end{array}$ \\
\hline $\begin{array}{l}\text { Building Description System } \\
\text { Building Design System } \\
\text { Parametric Building Model }\end{array}$ & $\begin{array}{l}\text { Bergin (2011): } \\
\text { termos encontrados na } \\
\text { investigação preliminar }\end{array}$ \\
\hline $\begin{array}{l}\text { Building Product Model } \\
\text { Integrated Product Model } \\
\text { Design Data Model } \\
\text { Computer-aided Architectural Design } \\
\text { Virtual Design and Construction } \\
\text { Integrated Design Model } \\
\text { Integrated Building Model }\end{array}$ & $\begin{array}{l}\text { termos encontrados na } \\
\text { investigação secundária }\end{array}$ \\
\hline
\end{tabular}

Esses termos tiveram seus significados verificados e validados de acordo com os termos históricos de referência, assim como em relação ao entendimento contemporâneo sobre o significado do BIM, segundo o protocolo de pesquisa apresentado a seguir.

\section{Método}

Para a condução da pesquisa foi adotado o método da Revisão Sistemática da Literatura (RSL) (Kitchenham, 2004). O protocolo estabelecido para orientar a pesquisa é apresentado na sequência.

As seguintes definições regem a RSL:

- Intervenção: 1) Quais foram as denominações que deram origem ao termo BIM, em que sequência e em que ordem temporal surgiram? 2) Quais foram os significados associados ao termo BIM? 3) Esse significado evoluiu? Caso afirmativo, como evoluiu?

- Controle: 1) termos extraídos por Bergin (2011) a partir de texto de Engelbart (1962), como referência histórica. 2) Definição contemporânea de BIM por Eastman et al. (2008) como referência de controle.

- População: Artigos científicos que passaram por revisão de pares e livros.

- Aplicação: Ao relacionar o sentido e as nuances do acrônimo BIM a seu contexto histórico de modo sistematizado, o presente trabalho pretende contribuir para que o significado de BIM possa ser melhor compreendido.

As seguintes bases bibliográficas foram utilizadas: (1) Web of Science (https://www.webofknowledge.com/); (2) ProQuest (http://www.proquest.com); (3) Scopus (www.scopus.com); e (4) Science Direct (www.sciencedirect.com).

Foram definidos os seguintes critérios de inclusão de fontes:

- Termos de busca: Termos de busca encontrados nas investigações preliminar e secundária, conforme apresentados na Tabela 2.

- Idioma: Inglês.

- Campos de busca: Título, resumo, palavras-chave e texto completo.

- Tipos de fonte: Livros, capítulos de livros, artigos em periódicos acadêmicos, dissertações e teses, artigos de conferência.

- Áreas temáticas e tipos de documentos: Os critérios de inclusão quanto à áreas temáticas e tipos de documento variaram de acordo com as opções fornecidas por cada base de dados, porém estiveram sempre dentro do escopo previsto na parte III do protocolo, seções "Campos de busca" e "tipos de fonte".

- Período: Entre 1962 (Engelbart, 1962) e 2016.

Foram definidos os seguintes critérios de exclusão de fontes:

- Tipos de documentos: Foram excluídas as patentes, revistas de caráter comercial (não-acadêmicas), brochuras, panfletos e outras mídias impressas não listadas nos critérios de inclusão.

- Áreas temáticas: Foram excluídos os trabalhos que não têm relação com Arquitetura, Engenharia ou Construção.

- Registros duplicados: Foram excluídos registros duplicados a respeito de uma mesma publicação. Esse processo foi feito em duas etapas: na primeira, foram 
excluídos os registros duplicados em uma mesma base. Posteriormente, foi feita a exclusão de registros entre bases diferentes.

A extração e filtragem das fontes para obtenção da amostra de estudo seguiu os seguintes passos:

- Aplicação dos filtros de inclusão em cada base de dados.

- Definição dos termos unificadores, para os casos em que as bases de dados exigem a entrada de variáveis semelhantes (porém, diferentes), para que se obtenha o mesmo resultado. Em uma determinada base, por exemplo, ao se procurar "Building Information model*", obtém-se variações do lema "model", ao posto que, em outra base, é preciso buscar por "Model", Modeling" e "Modelling" para se ter resultados equivalentes.

- Identificação de artigos que aparecem duplicados em uma mesma base de dados, por possuírem: 1) mesmo Termo Unificador, DOI, Título, Nome dos autores, Tipo de Publicação e Tipo de Documento, 2) na falta de DOI, além dos critérios acima, foram comparados os campos Volume, Página Inicial, Página Final e Número de Páginas; e 3) qualquer diferença entre qualquer um desses campos fez com que os registros em exame permanecessem na base de dados.

- Identificação da base de dados principal (no âmbito desta pesquisa) e eliminação das duplicações entre as bases de dados. Os critérios que permitiram a definição da base de dados principal, de onde foi feita a extração de significados dos termos, bem como a ordem de prioridade das outras bases para que fosse feito o processo de eliminação de seus duplicados, estão na Tabela 2.

Tabela 2: Características das bases de dados e ordem de prioridade na remoção de duplicações. Fonte: Autores.

\begin{tabular}{|c|c|c|c|c|}
\hline Características desejadas & 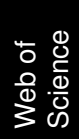 & 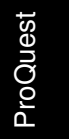 & 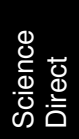 & $\begin{array}{l}\text { 号 } \\
\text { 응 } \\
\text { ભ }\end{array}$ \\
\hline $\begin{array}{l}\text { Permite pesquisa em textos } \\
\text { completos? }\end{array}$ & Não & Sim & Sim & Sim \\
\hline Inclui livros? & Não & Sim & Sim & Sim \\
\hline Permite exportar metadados? & Sim & Sim & Sim & Sim \\
\hline $\begin{array}{l}\text { Exibe o número de citações por } \\
\text { artigo? }\end{array}$ & Sim & Não & Não & Sim \\
\hline $\begin{array}{l}\text { Ordem de prioridade na remoção de } \\
\text { duplicados }\end{array}$ & $1^{\mathrm{a}}$ & $2^{\mathrm{a}}$ & $3^{\mathrm{a}}$ & $4^{\mathrm{a}}$ \\
\hline
\end{tabular}

Para a identificação de significados atribuídos a cada termo, foram extraídos dos textos o significado original (na publicação mais antiga que continha uma definição do termo) e o significado na publicação mais citada, o que é uma indicação indireta de aceitação da definição daquele termo. Os significados foram obtidos a partir da leitura dos artigos, identificando-se trechos onde os autores atribuíram uma definição ou descrição do termo. Esse processo de identificação dos significados permitiu a validação dos termos que tiveram suas frequências de uso no tempo calculados.

O resultado do processo busca das fontes, inclusão, exclusão e eliminação de duplicados entre as bases pesquisadas é apresentado na Tabela 3, o que leva à amostra utilizada nas análises.

Tabela 3: Amostra final em quantidade de fontes bibliográficas por base e termo unificador. Fonte: Autores.

\begin{tabular}{|c|c|c|c|c|c|}
\hline Termo Unificador & 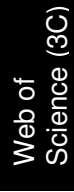 & 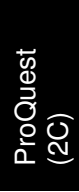 & 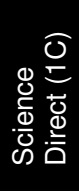 & $\begin{array}{l}\text { 号 } \\
\text { 응 } \\
\text { ळ }\end{array}$ & $\begin{array}{l}\frac{1}{\mathbb{L}} \\
\stackrel{\circ}{\circ}\end{array}$ \\
\hline Building Description System & 0 & 17 & 20 & 28 & 65 \\
\hline Building Design System & 8 & 35 & 76 & 107 & 226 \\
\hline Building Information Model & 491 & 717 & 846 & 3512 & 5566 \\
\hline Building Product Model $^{*}$ & 22 & 163 & 19 & 452 & 656 \\
\hline $\begin{array}{l}\text { Computer-aided Architectural } \\
\text { Design }\end{array}$ & 65 & 213 & 177 & 1730 & 2185 \\
\hline Design Data Model ${ }^{*}$ & 12 & 13 & 33 & 46 & 104 \\
\hline Integrated Building Model ${ }^{*}$ & 1 & 28 & 13 & 11 & 53 \\
\hline Integrated Design Model* & 10 & 12 & 34 & 49 & 105 \\
\hline Integrated Product model ${ }^{*}$ & 23 & 5 & 20 & 205 & 253 \\
\hline Parametric Building Model* & 3 & 6 & 9 & 34 & 52 \\
\hline Virtual Design and Construction & 4 & 68 & 31 & 286 & 389 \\
\hline Total & 638 & 1277 & 1278 & 6460 & 9654 \\
\hline
\end{tabular}

\section{O significado dos termos}

A seguir são apresentados os termos pesquisados com a identificação de seus significados, de acordo com o protocolo da pesquisa.

\section{Computer-aided Architectural Design (1974)}

A definição mais antiga encontrada sobre o termo Computeraided Architectural Design (CAAD) nesta pesquisa está em Eastman (1974). O autor afirma que, dentre os principais aspectos que definem CAAD, estão automated design, reading and interpretation of architectural drawings e information structures for achitectural information.

$\mathrm{O}$ artigo mais citado que contém uma definição sobre CAAD, dentro da pesquisa, é de Heylighen e Neuckermans (2000). Os autores afirmam que o termo, até aquela época, havia atravessado gerações e diferentes visões filosóficas e que, entre o começo e a metade da década de 90, a vertente filosófica mais popular do CAAD era o Case-Based Design (CBD), metodologia computacional de desenvolvimento de projetos baseada em inteligência artificial aplicada a um repertório prévio de soluções de projeto (Watson e Perera, 1997). Percebe-se, portanto, que em 24 anos o significado atribuído ao CAAD sofreu alterações e se distanciou do significado original, o qual apresentava muita similaridade com o termo contemporâneo relacionado a BIM (que era o Building Description System).

\section{Building Design System (1975)}

No artigo de Lesniak, Grodzki e Wintarski (1975), o mais antigo que contém o termo, Building Design System, é 
definido como sendo um sistema computacional de projetos de edifícios adequado à construção sistematizada e industrializada. No artigo mais citado que contém o termo, de Augenbroe (1992), um Intelligent Integrated Building Data System (IIDBS) é definido como um sistema orientado a objetos capaz de oferecer uma descrição completa da edificação por meio de um building data model conceitual que permite a troca de dados a partir de uma especificação de padrão neutro (à época, o STEP era a opção sugerida pelo autor).

\section{Building Description System (1976)}

A primeira definição do termo Building Description System (BDS) aparece em Eastman (1976). Esse artigo é também o que contém mais citações dentre aqueles que apresentam o termo. O autor afirma que os BDS são programas constituídos por grandes bancos de dados que têm o potencial de substituir desenhos como documentos para a construção. Além disso, afirma o autor, esse modo sistemático de descrição de edifícios poderia permitir análises visuais, coordenação, quantitativos, avaliação de códigos de edificações e até mesmo fabricação automatizada de componentes.

\section{Integrated Building Model (1980)}

Eastman (1980) publica o primeiro (e único, no âmbito desta pesquisa, dentre os mais citados) artigo que traz a definição do termo Integrated Building Model. O autor deu o nome de Integrated Building Model ao resultado de seu trabalho, formado por um sistema de equipamentos (hardware) que dava suporte a um banco de dados com as informações do modelo. A solução realizada por Eastman deveria ser capaz de fornecer e armazenar informações para programas de análise de critérios funcionais, análise estrutural e térmica, orçamento e modelagem financeira.

\section{Design Data Model (1982)}

O primeiro registro do termo Design Data Model encontrado na pesquisa está em um artigo de Foisseau e Valette (1982), porém não foi possível encontrar o texto original para consulta. O primeiro artigo (e também o mais citado) encontrado com uma clara definição do termo é de Wang e Naaji (2004). O trabalho que apresentam, à época, é a proposição de uma nova estrutura de design data model. Para conceituar o termo, os autores fazem uma lista de requisitos que definem o que é um object-oriented CAD data model, com base em Spooner (1991).

\section{Integrated Product Model (1988)}

$\mathrm{O}$ artigo mais antigo e que traz a definição desse termo, de acordo com o protocolo desta pesquisa, é de Arai e Iwata (1988). Wölkl e Shea (2009) escrevem o artigo com mais citações com uma definição para esse termo. Para eles, um Integrated Product Model deve ser estruturado de modo a poder representar e conectar requisitos, funções e princípios de um modelo, suportando múltiplos níveis de abstração e detalhe, o qual possa ser reutilizado total ou parcialmente e que possibilite a criação de representações de si próprio, para facilitar o trabalho dos projetistas.

\section{Building Product Model (1989)}

Björk (1989) apresenta dois significados para o termo Building Product Model (BPM), nesse que é o artigo mais antigo relacionado à busca. Nele há uma descrição técnica afirmando que um BPM deve conter objetos e atributos relacionados, com a função de descrever uma edificação, além de uma descrição relacionada a processos, a fim de que o modelo pode ser expandido e alterado pelos diversos participantes para atender às demandas de todo o ciclo de vida do projeto e obra. No artigo mais citado, de Howard e Björk (2008), o termo é definido como identificador de um método de se fazer a descrição digital de uma edificação utilizando objetos com classificação estruturada. Esses autores comentam que, recentemente (para a época), vendedores de software cunharam um novo termo - o Building Information Model (BIM) - para se referir essencialmente à mesma coisa.

\section{Building Information Model (1992)}

O termo Building Information Model (BIM) aparece pela primeira vez, nesta pesquisa, entre as palavras-chaves do artigo de Nederveen e Tolman (1992). A definição do termo entre os artigos mais citados e que estão no escopo desta pesquisa é de Succar (2009), que usa um trecho de Penttilä (2006). Succar afirma que BIM é um conjunto de políticas, processos e tecnologias que, interagindo, geram uma metodologia para a gestão, em formato digital, do projeto de uma edificação e de seus dados, durante todo seu ciclo de vida. Verificou-se que essa foi a primeira definição encontrada em que um autor utiliza palavras como policies, process e manage na elaboração do significado, afastando-o, nesse caso, de uma conotação essencialmente computacional ou tecnológica. Foi constatado, também, que Succar (2009) foi o artigo com mais citações dentre todas as categorias (ou seja, entre todos os termos) investigadas nesta pesquisa.

\section{Integrated Design Model (1992)}

O artigo mais antigo que contém uma definição para o termo Integrated Design Model é de Huebel, Ruland e Siepmann (1992). Em Braukhane e Quantius (2011), o artigo com mais citações na pesquisa que contém a definição do termo, os autores descrevem o processo adotado pela Agência Espacial Europeia, chamado Integrated Design Model, que consiste em um conjunto de arquivos de Excel padronizados que recebem dados de diferentes ferramentas de projeto, entre outros sistemas, com o objetivo de permitir a colaboração transcontinental, evitando redundância nas informações.

\section{Parametric Building Model (2004)}

Salazar, Polat e Almeida (2004) apresentam a definição mais antiga encontrada sobre o termo Parametric Building Model no escopo desta pesquisa. No artigo mais citado que contém definição sobre o termo, Lee, Sacks e Eastman (2006) afirmam que, dentre os vários sistemas que permitem o 
trabalho em BIM, um sistema de modelagem paramétrica, rico em informações e em 3D, tem a propriedade de manter a validade das informações produzidas, sendo crucial para a revisão e reutilização da informação da construção a partir da imposição de restrições geométricas e outras regras, enquanto o modelo da construção passa por revisões.

\section{Virtual Design and Construction (2004)}

Kam e Fischer (2004) são autores do artigo mais antigo que contém uma definição sobre o termo Virtual Design and Construction. Afirmam que a modelagem e simulação por meio de projeto e construção virtual permite alternar rapidamente entre escolhas de projeto, prever e quantificar 0 comportamento dos objetos com o objetivo de avaliar alternativas antes de tomar decisões importantes. Para tanto, na interpretação dos autores, a VDC faz uso de modelagem orientada a processos (POP), modelagem do produto (product model) e simulação da construção no tempo (4D). Com relação à publicação com mais citações que contêm significado atribuído ao termo, Sacks el al. (2010), em artigo que demonstra as possibilidades de integração entre BIM e lean construction, afirmam que o conceito de VDC pode ser tomado como uma representação do BIM, ou de aspectos do $\mathrm{BIM}$, dadas as similaridades nos princípios e tecnologias que os sustentam.

\section{Os termos no tempo}

A quantidade de fontes bibliográficas que continham os termos unificadores (Tabela 3) foi distribuída na linha do tempo em forma gráfica (Figuras 1 a 4) e tabular (Tabela 4). Os termos estão ordenados de acordo com a data da primeira citação de cada um deles.

Na Figura 1, a quantidade de publicações por termo unificador é apresentada para o período de 1971 a 2016.

As Figuras 2 a 4 foram produzidas para se ter um melhor entendimento visual de como se deu a frequência de uso dos termos estudados. A grande quantidade de resultados apresentados para o termo Building Information Modeling, na Figura 1, tornou difícil a comparação e análise dos demais termos com resultados de menor valor por ano, e foi o fator que motivou a criação das demais figuras.

A Tabela 4, por conseguinte, apresenta os números que dão origem às Figuras 1 a 4 ; neste caso, optou-se por agrupar os resultados em períodos de 5 anos.

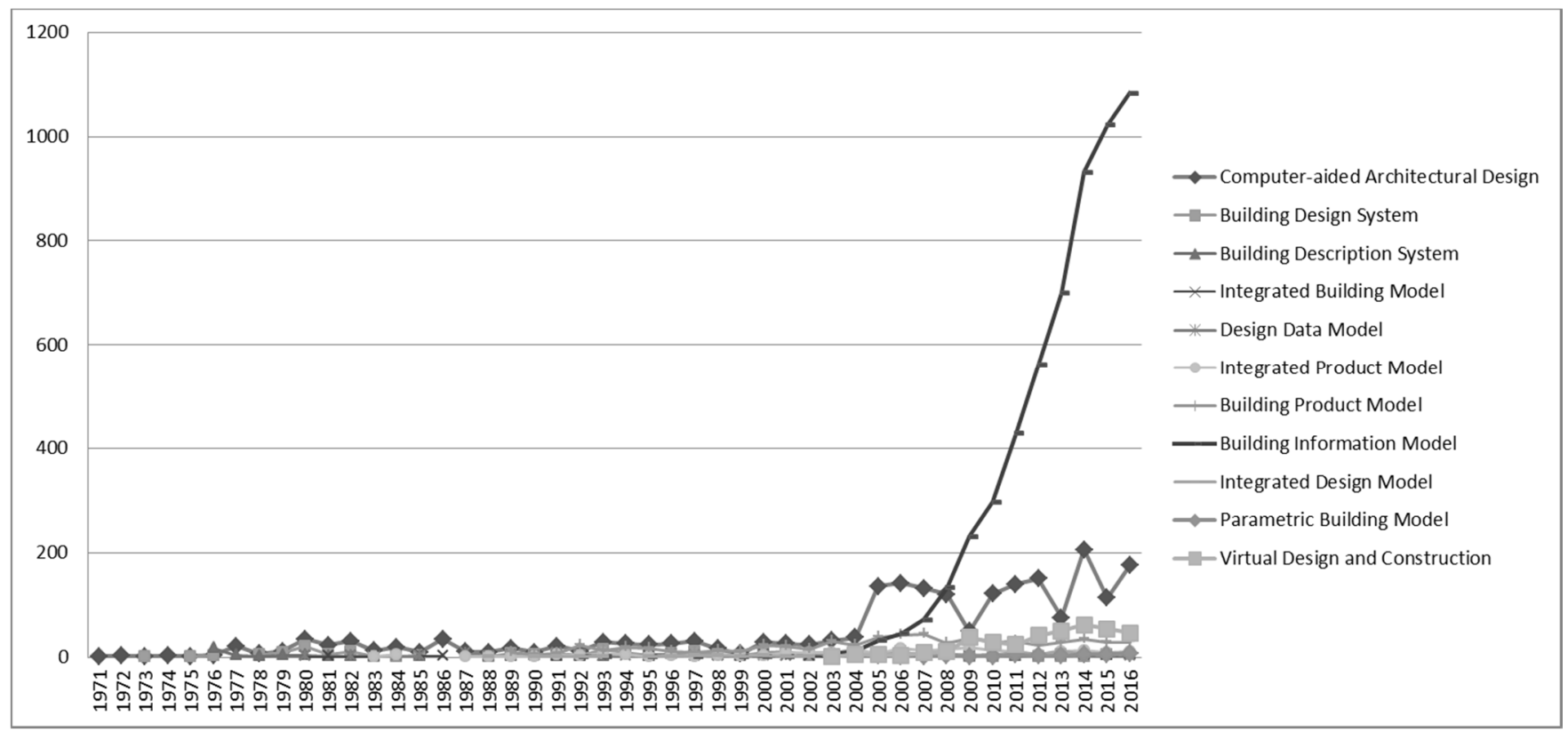

Figura 1: Quantidade de publicações que apresentam os termos que guardam relação de significado com o significado do termo BIM de referência (Eastman et al., 2008) ao longo dos anos. Fonte: Autores. 


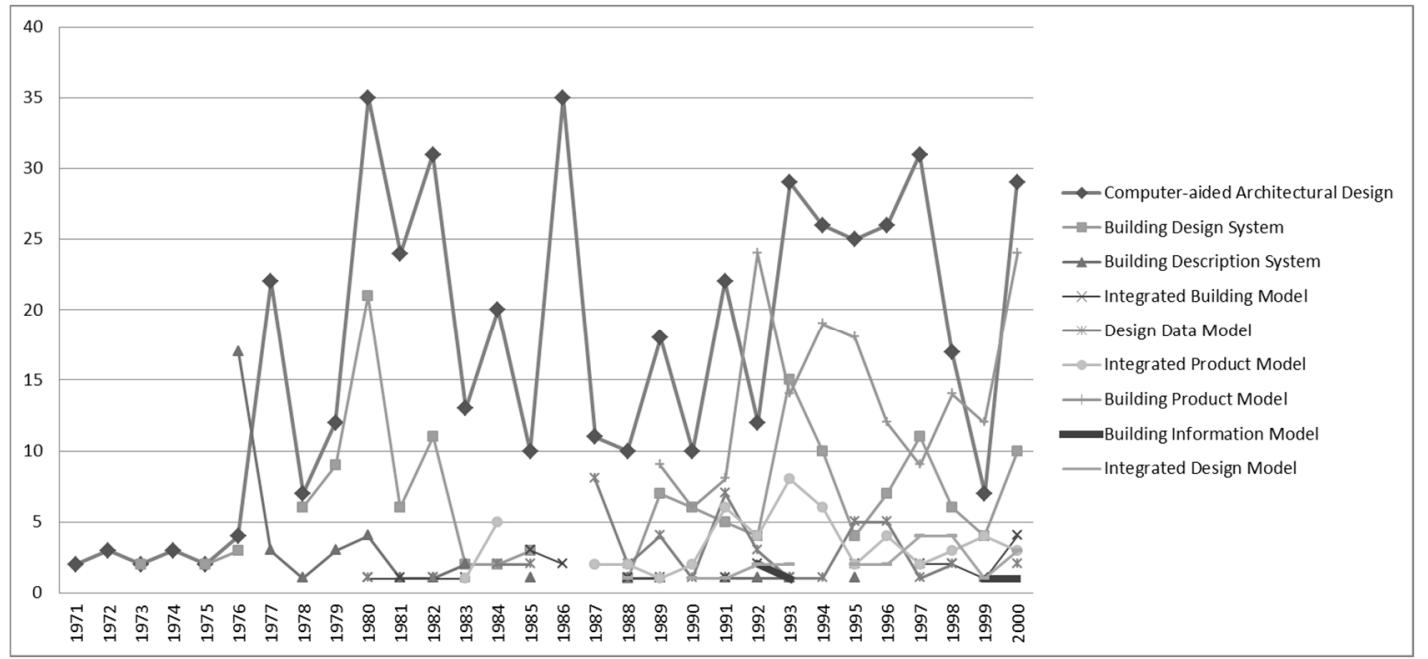

Figura 2: Resultados da pesquisa até 2000. Fonte: Autores.

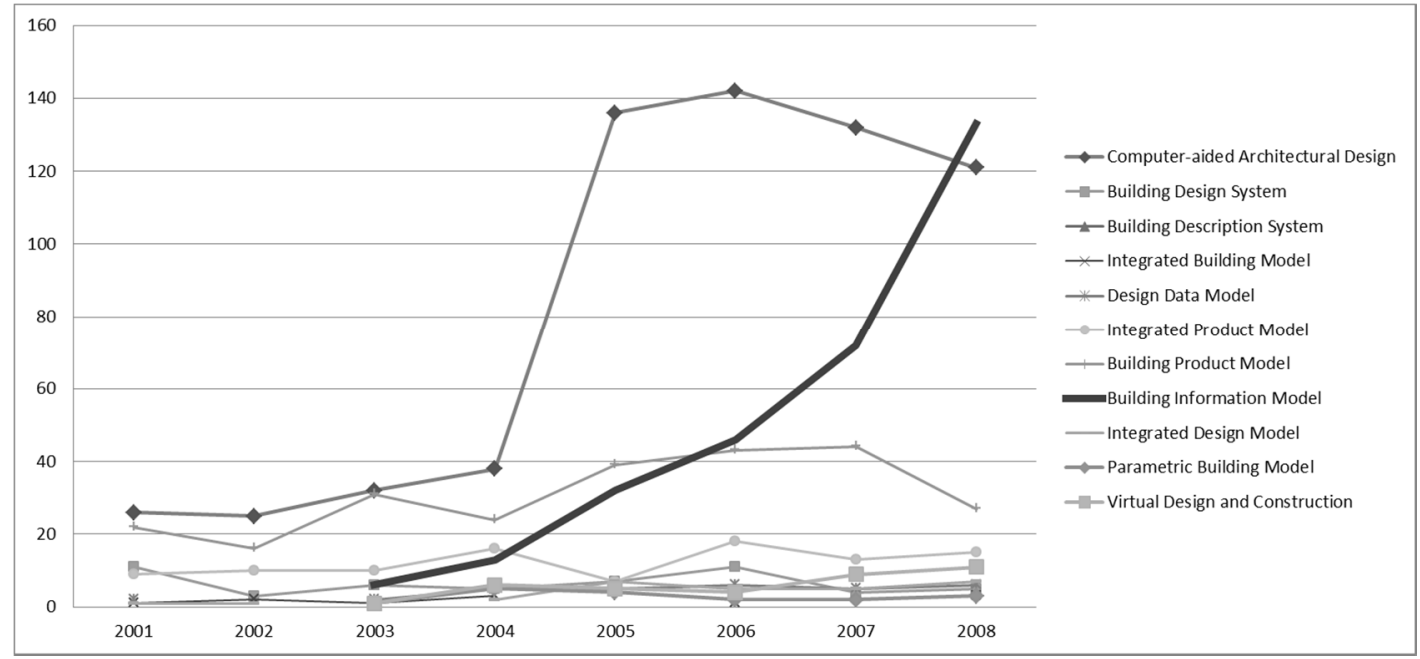

Figura 3: Resultados da pesquisa entre 2001 e 2008. Fonte: Autores.

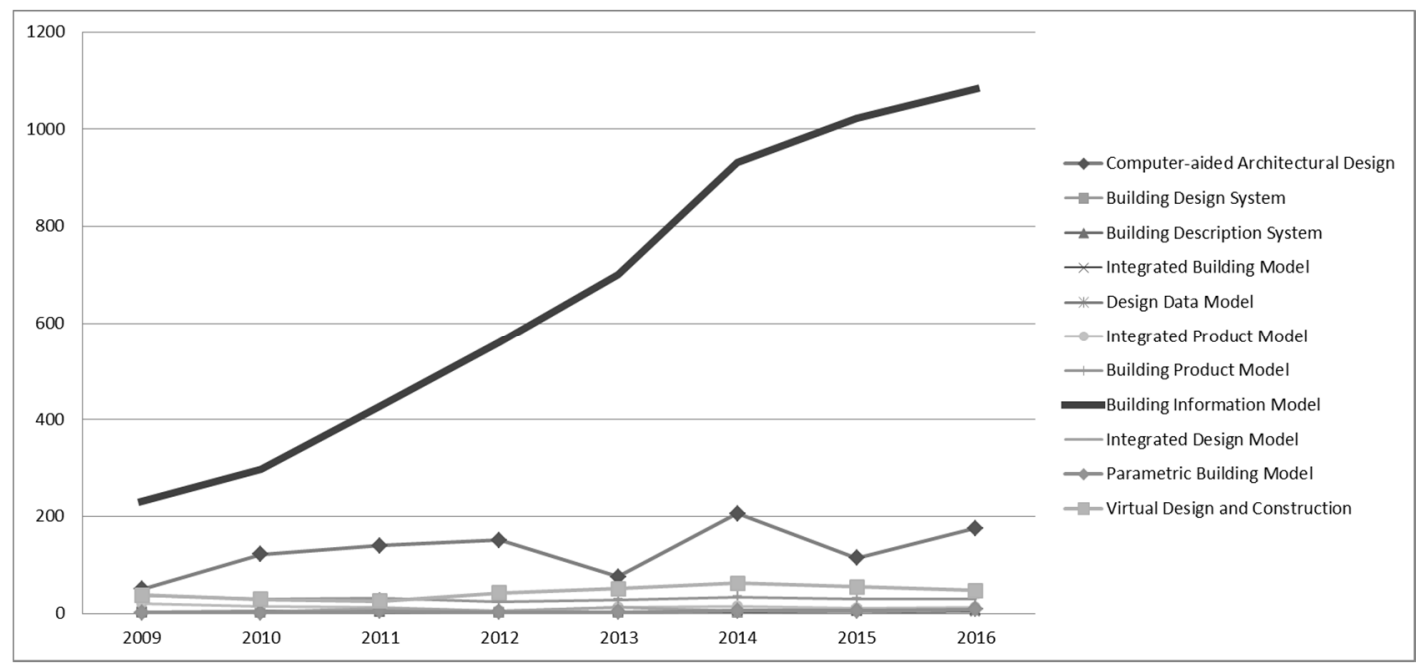

Figura 4: Resultados da pesquisa entre 2009 e 2016. Fonte: Autores. 
Tabela 4: Quantidade de publicações que apresentam os termos, agrupados em períodos de 5 anos. Fonte: Autores.

\begin{tabular}{|c|c|c|c|c|c|c|c|c|c|c|}
\hline Termo Unificador & $\begin{array}{l}1971- \\
1975\end{array}$ & $\begin{array}{l}1976- \\
1980\end{array}$ & $\begin{array}{l}1981- \\
1985\end{array}$ & $\begin{array}{l}1986- \\
1990\end{array}$ & $\begin{array}{l}1991- \\
1995\end{array}$ & $\begin{array}{l}1996- \\
2000\end{array}$ & $\begin{array}{l}2001- \\
2005\end{array}$ & $\begin{array}{l}2006- \\
2010\end{array}$ & $\begin{array}{l}2011- \\
2016\end{array}$ & $\begin{array}{l}\text { Total } \\
\text { Geral }\end{array}$ \\
\hline Computer-aided Architectural Design (1974) & 12 & 80 & 98 & 84 & 114 & 110 & 257 & 567 & 863 & 2185 \\
\hline Building Design System (1975) & 4 & 39 & 24 & 14 & 38 & 38 & 32 & 23 & 14 & 226 \\
\hline Building Description System (1976) & & 28 & 5 & 2 & 4 & & 1 & 1 & 24 & 65 \\
\hline Integrated Building Model (1980) & & 1 & 6 & 4 & 6 & 9 & 7 & 6 & 14 & 53 \\
\hline Design Data Model (1982) & & 1 & 5 & 15 & 17 & 10 & 14 & 26 & 16 & 104 \\
\hline Integrated Product Model (1988) & & & 6 & 7 & 26 & 16 & 52 & 80 & 66 & 253 \\
\hline Building Product Model (1989) & & & & 15 & 83 & 71 & 132 & 180 & 175 & 656 \\
\hline Building Information Model (1992) & & & & & 4 & 3 & 52 & 781 & 4726 & 5566 \\
\hline Integrated Design Model (1992) & & & & 2 & 7 & 14 & 11 & 24 & 47 & 105 \\
\hline Parametric Building Model (2004) & & & & & & & 10 & 11 & 31 & 52 \\
\hline Virtual Design and Construction (2004) & & & & & & & 12 & 91 & 282 & 385 \\
\hline Total Geral & 16 & 149 & 144 & 143 & 299 & 271 & 580 & 1790 & 6258 & 9650 \\
\hline
\end{tabular}

\section{Conclusão}

O objetivo deste estudo foi registrar a evolução do termo BIM ao longo de sua história, assim como seu significado. As questões que guiaram este registro foram relativas às denominações que deram origem ao termo BIM, o aparecimento desses termos e sua evolução ao longo do tempo, assim como seus significados associados.

Quanto às denominações, foi possível identificar os termos precursores ao BIM: Building Design System (Lesniak, Grodzki e Wintarski 1975), Building Description System (Eastman, 1976), Integrated Building Model (Eastman, 1980), Design Data Model (Encarnacao e Krause, 1982) e Integrated Product Model (Arai e Iwata, 1988). A Tabela 4 sustenta visualmente esta afirmação, pois explicita a ordem temporal de surgimento dos termos estudados. O termo Building Product Model (Björk, 1992) continua em uso em menor escala. Percebe-se que o termo Building Information Model ganha força a partir de 2004 e se torna o mais utilizado entre 2008 e 2009; hoje, seu uso é predominante (Figura 1). Foi percebido que o uso de Computer-aided Architectural Design teve um grande crescimento em 2005 e que o número absoluto de artigos que usam esse termo, por ano, não sofreu grande aumento entre 2005 e 2016 . Entre os termos mais novos que o Building Information Model, o Virtual Design and Construction se destaca dos demais (Integrated Design Model e Parametric Building Model), quando se trata do crescimento de sua adoção por parte da academia no período entre 2001 e 2016.

Foram identificados dois termos com força de expressão (Tabela 4) que inicialmente tinham semelhança em significado, mas se diferenciaram com o tempo: Computeraided Architectural Design e Building Information Modeling. Heylighen e Neuckermans (2000) e Succar (2009), respectivamente, se destacam pela definição corrente desses termos. Verificou-se, também, que o termo Virtual Design and Construction, segundo Sacks et al. (2010), guarda semelhança de significado com o termo Building Information Modeling.
Os termos precursores do BIM (Building Design System, Building Description System, Integrated Building Model, Design Data Model, Integrated Product Model) apresentam significados semelhantes, em que a ênfase é dada nas tecnologias que dão suporte à produção de projeto em computador, com destaque para a modelagem orientada a objeto. Apenas Succar (2009), na amostra estudada, em sintonia com o termo BIM de controle (Eastman et al., 2008), confere-lhe um sentido que ultrapassa os limites da técnica computacional, atribuindo-lhe um significado mais amplo, que tem relação com os processos de produção de projeto e construção. Percebe-se, assim, uma evolução no significado atribuído ao termo BIM.

A RSL desenvolvida deu suporte à investigação, análise e validação dos termos procurados e apresentados neste estudo e suas relações de significado com os termos de referência. Todos os termos apresentados nas tabelas e figuras deste artigo tiveram seus significados confirmados e validados dentro das regras estabelecidas pelo protocolo de pesquisa, ou seja, todos eles possuem relação similar de significado com os termos de referência, tanto históricos (Engelbart, 1962), quanto de controle (Eastman et al., 2008).

Alguns termos preliminares, submetidos ao mesmo processo de análise, foram eliminados, uma vez que não foi possível obter resultados aceitáveis de acordo com os parâmetros de qualidade estabelecidos pelo protocolo. $O$ fato de que alguns termos foram confirmados pelo método, enquanto outros não o foram, pode ser um indício de que o método apresentado poderá ser utilizado para verificar outros termos em outra oportunidade, como forma de ampliar ou atualizar o presente estudo.

Entre as limitações da pesquisa, foi possível observar que alguns dos termos originalmente procurados foram eliminados na fase preliminar porque os artigos que os definem não estão listados na base Scopus, definida como referência principalmente por permitir buscas em textos completos e ter índice de citações por artigo. Outra limitação encontrada tem relação com a dificuldade de acesso a 
publicações muito antigas, em que não existem versões eletrônicas publicadas para consulta.

Quanto à interpretação dos resultados, poderiam ser feitos estudos com relação à importância relativa dos termos encontrados, aferindo a proporção de cada um em relação ao total, ao longo do tempo. Também poderiam ser realizadas análises mais aprofundadas sobre os significados atribuídos a cada um dos termos ao longo dos anos, identificando-se em que momentos suas definições se aproximam ou se afastam.

Com relação à lista de termos procurados, o ponto de partida poderia ser ampliado para aperfeiçoamento do protocolo. Outro melhoramento seria a aplicação de processos para a extração de significados a partir de uma grande quantidade de textos, como forma de aproveitar as informações das outras bases de dados (e não apenas da Scopus) nas etapas finais da pesquisa.

\section{Referências}

Arai, E., \& Iwata, K. (1988). Product design logic for an intelligent product modelling system. Robotics and Computer Integrated Manufacturing, 4(3-4), 499-510. doi:10.1016/07365845(88)90022-1

Augenbroe, G. (1992). Integrated building performance evaluation in the early design stages. Building and Environment, 27(2), 149161. doi:10.1016/0360-1323(92)90019-L

Bergin, M. S. (2011). History of BIM. Retrieved from http://www.architectureresearchlab.com/arl/2011/08/21/bimhistory/

Björk, B. C. (1989). Basic structure of a proposed building product model. Computer-Aided Design, 21(2), 71-78. doi:10.1016/00104485(89)90141-3

Braukhane, A., \& Quantius, D. (2011). Interactions in space systems design within a Concurrent Engineering facility. Paper presented at the Proceedings of the 2011 International Conference on Collaboration Technologies and Systems, CTS 2011.

Eastman, C. (1976). General purpose building description systems. Computer-Aided Design, 8(1), 17-26. doi:10.1016/00104485(76)90005-1

Eastman, C., Teicholz, P., Sacks, R., Liston, K., \& Handbook, B. (2008). A Guide to Building Information Modeling for Owners, Managers, Architects, Engineers, Contractors, and Fabricators. In: John Wiley and Sons, Hoboken, NJ.

Eastman, C. M. (1974). Through the looking glass: why no wonderland. Computer applications to architecture in the USA. Computer-Aided Design, 6(3), 119-124. doi:10.1016/00104485(74)90042-6

Eastman, C. M. (1980). Prototype integrated building model. Computer-Aided Design, 12(3), 115-119. doi:10.1016/00104485(80)90003-2

Engelbart, D. C. (2001). Augmenting human intellect: a conceptual framework (1962). PACKER, Randall and JORDAN, Ken. Multimedia. From Wagner to Virtual Reality. New York: WW Norton \& Company, 64-90.

Foisseau, J., \& Valette, F. R. (1982). A computer aided design data model: FLOREAL. File Structures and Data Bases for CAD, 315334.

Heylighen, A., \& Neuckermans, H. (2001). A case base of case-based design tools for architecture. CAD Computer Aided Design, 33(14), 1111-1122. doi:10.1016/S0010-4485(01)00055-0
Howard, R., \& Björk, B. C. (2008). Building information modelling Experts' views on standardisation and industry deployment. Advanced Engineering Informatics, 22(2), 271-280. doi:10.1016/j.aei.2007.03.001

Huebel, C., Ruland, D., \& Siepmann, E. (1992). On modeling integrated design environments. Paper presented at the European Design Automation Conference.

Kam, C., \& Fischer, M. (2004). Capitalizing on early project decisionmaking opportunities to improve facility design, construction, and life-cycle performance - POP, PM4D, and decision dashboard approaches. Automation in Construction, 13(1), 53-65. doi:10.1016/j.autcon.2003.08.004

Kitchenham, B. (2004). Procedures for performing systematic reviews. Keele, UK, Keele University, 33(2004), 1-26.

Lee, G., Sacks, R., \& Eastman, C. M. (2006). Specifying parametric building object behavior (BOB) for a building information modeling system. Automation in Construction, 15(6), 758-776. doi:10.1016/j.autcon.2005.09.009

Lesniak, Z. K., Grodzki, Z., \& Winiarski, M. S. (1975). Optimisation of industrialised building systems. Building Science, 10(3), 169-175. doi:10.1016/0007-3628(75)90015-8

Penttilä, H. (2006). Describing the changes in architectural information technology to understand design complexity and free-form architectural expression. Electronic Journal of Information Technology in Construction, 11, 395-408.

Sacks, R., Koskela, L., Dave, B. A., \& Owen, R. (2010). Interaction of lean and building information modeling in construction. Journal of Construction Engineering and Management, 136(9), 968-980. doi:10.1061/(ASCE)CO.1943-7862.0000203

Salazar, G. F., Polat, I. H., \& Almeida, J. C. (2004). The Role of the 3D Parametric Building Model in the Future Education and Practice of Civil Engineering and Construction. In Towards a Vision for Information Technology in Civil Engineering (pp. 1-14).

Spooner, D. L. (1991). Towards an Object-Oriented Data Model for a Mechanical CAD Database System. In K. R. Dittrich, U. Dayal, \& A. P. Buchmann (Eds.), On Object-Oriented Database Systems (pp. 189-205). Berlin, Heidelberg: Springer Berlin Heidelberg.

Succar, B. (2009). Building information modelling framework: A research and delivery foundation for industry stakeholders. Automation in Construction, 18(3), 357-375. doi:10.1016/j.autcon.2008.10.003

van Nederveen, G. A., \& Tolman, F. P. (1992). Modelling multiple views on buildings. Automation in Construction, 1(3), 215-224. doi:10.1016/0926-5805(92)90014-B

Wang, Y., \& Nnaji, B. O. (2004). UL-PML: Constraint-enabled distributed product data model. International Journal of Production Research, 42(17), doi: $10.1080 / 002075403410001708443$ 3743-3763.

Watson, I., \& Perera, S. (1997). Case-based design: A review and analysis of building design applications. Artificial Intelligence for Engineering Design, Analysis and Manufacturing: AIEDAM, 11(1), 59-87.

Wölkl, S., \& Shea, K. (2009). A computational product model for conceptual design using sysml. Paper presented at the Proceedings of the ASME Design Engineering Technical Conference. 